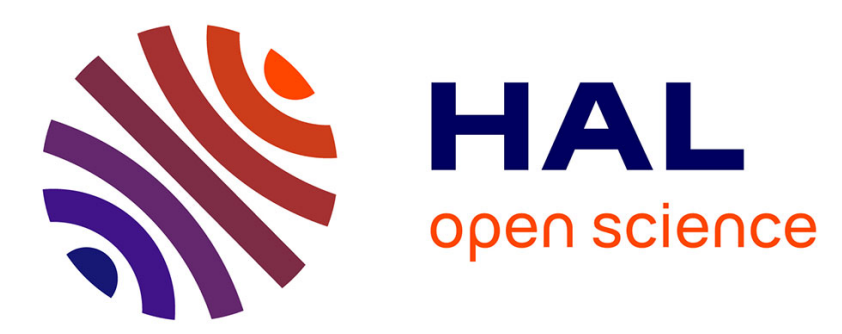

\title{
Dynamics of core merging after a mega-impact with applications to Mars' early dynamo
}

\author{
J. Monteux, A.M. M Jellinek, C.L. L Johnson
}

\section{To cite this version:}

J. Monteux, A.M. M Jellinek, C.L. L Johnson. Dynamics of core merging after a mega-impact with applications to Mars' early dynamo. Icarus, 2013, 226 (1), pp.20-32. 10.1016/j.icarus.2013.05.008 . hal-01636055

\section{HAL Id: hal-01636055 \\ https://hal.uca.fr/hal-01636055}

Submitted on 11 Jan 2018

HAL is a multi-disciplinary open access archive for the deposit and dissemination of scientific research documents, whether they are published or not. The documents may come from teaching and research institutions in France or abroad, or from public or private research centers.
L'archive ouverte pluridisciplinaire HAL, est destinée au dépôt et à la diffusion de documents scientifiques de niveau recherche, publiés ou non, émanant des établissements d'enseignement et de recherche français ou étrangers, des laboratoires publics ou privés. 


\section{Dynamics of core merging after a martian mega-impact}

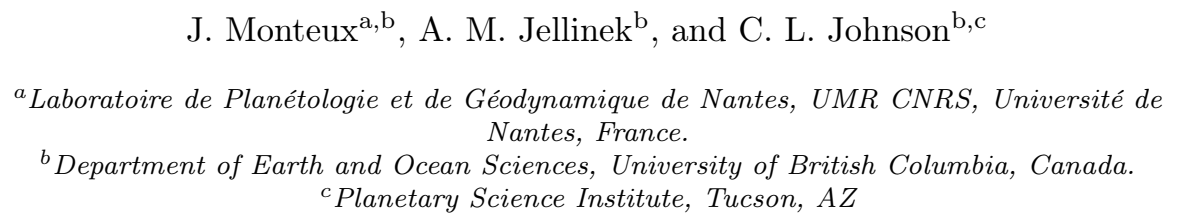

7 Abstract

A number of geophysical arguments point out the possibility that a giant impact occurring within the first 500 Myr of the martian history could be responsible for the dichotomy between the northern lowlands and the southern highlands. This giant impact may also have important consequences on the initiation or cessation of the transient martian dynamo. At the time of the impact both the impactor and Mars were differentiated. Hence, an important volume of metallic material from the impactor probably merged with the martian preimpact core. In this study, we characterize this core merging dynamics assuming that the impactor's core sank as a single diapir within the martian mantle and we obtain characteristic timescales of the merging time between the two cores as a function of the rheology and the size of the sinking diapir. For large impacts, the coalescence time should take less than $1 \mathrm{Myr}$ even for a very viscous mantle and this time linearly decreases with decreasing mantle viscosity. We also measure the influence of the impact heating and of the viscous heating associated with the core merging on the martian thermal state as a function of the rheology and size of the impactor. Finally, we discuss the influence of such a giant impact on the magnetic history of Mars. We show that, assuming perfect thermal mixing between the hot merging core and the planetary core, dynamo activity might be favoured by large impacts, low mantle viscosity and a thin thermal boundary layer below the CMB. Considering that dynamo longevity depends on 
how long it takes to remove the added thermal anomaly, post-impact dynamo can eventually last several hundred Myrs.

\& Key words: meteoritical impacts; numerical modeling; mega-impacts; Mars;

- dynamo. 


\section{Introduction}

Mega impacts probably played an important role in the late history of terrestrial planetary accretion. A Mars-size impact can, for example, explain the Earth-Moon system (Hartmann and Davis, 1975) and a large impact removing part of the silicate mantle is a hypothesis for the high iron/silicate ratio on Mercury (Smith, 1979; Benz et al., 1988). Among the hypotheses for the origin of the martian dichotomy (Elkins-Tanton et al., 2003; Roberts and Zhong, 2006), an exogenic origin by a mega impact that displaced crustal material from the northern to the southern hemisphere seems to be the most plausible candidate (Wilhelms and Squyres, 1984; Nimmo et al., 2008; Andrews-Hanna et al., 2008; Marinova et al., 2008, 2011). Models for the martian impact suggest that the impactor was 200 to $1300 \mathrm{~km}$ in radius and hit the planet with a speed comparable to or larger than the martian escape velocity (i.e. $v_{i m p}>5 \mathrm{~km} . \mathrm{s}^{-1}$ ) within the first 500 Myr of Martian history (Frey, 2006).

In addition to a history of large impacts, Earth, Mercury and Mars have, or have had, an internally generated magnetic field. Mercury and the Earth have active core dynamos, whereas Mars had only a transient internally generated magnetic field (Acuña et al., 1999; Hood et al., 2003; Lillis et al., 2008a) that ceased by around 4.0 Ga (Acuña et al., 1999; Johnson and Phillips, 2005; Lillis et al., 2008b). The timing of the initiation of the martian dynamo is difficult to constrain and strongly depends on the differentiation processes that occurred during the first million years of the martian history (Monteux et al., 2011). The cessation of the martian dynamo is also still currently debated. Recent models have measured the influence of large impacts on the dynamo generation process and in particular on the cessation of core convection induced by the reduction of the core-mantle boundary (CMB) heat-flux (Roberts et al., 2009; Watters et al., 2009; Arkani-Hamed and Olson, 2010; Roberts and Arkani-Hamed, 2012). 
37 However, recent models show that the thermal anomaly induced by a large

38 impact impact and the formation of a hot molten iron layer from the impactor's

39 core cumulating at the CMB can also favor dynamo generation (Reese et al.,

$40 \quad 2010)$.

41 At the time of the proposed giant impact, both Mars and the impactor were

42 probably differentiated (Yoshino et al., 2003). Models suggest that although

43 some material was ejected far from Mars, the majority of the mass of the im-

44 pactor's core was retained within Mars and merged with the pre-impact Martian

45 core (Canup, 2004) (Marinova does not consider the impactor's core). The aim

46 of this study is to characterize the dynamics of core merging as a result of a

47 diapiric descent of molten iron (Monteux et al., 2009) on a Mars-size planet. In

48 addition, we investigate how the processes of impact and core merging might in-

49 fluence the thermal regime of Mars' core and, in turn, magnetic field generation

so (cf., Monteux et al. (2011)).

51 2. Thermo-chemical state before the martian mega-impact

52 2.1. Pre-impact interior of Mars

53 The initial structure and thermal state of a growing planet depends particu-

54 larly on the characteristics of its accretion from chondritic material (Safronov,

55 1978; Kaula, 1979; Agee, 1997). During accretion, heating driven by a combina-

56 tion of the dissipation of impact energy and the decay of short lived radionuclides

${ }_{57}$ such as ${ }^{26} \mathrm{Al}$ and/or ${ }^{60} \mathrm{Fe}$ (Yoshino et al., 2003; Monteux et al., 2007) increases

58 the mean internal temperature and gives rise to a radial temperature gradient

59 that depends on the accretion rate relative to the rate of radiative cooling to

6o space (Kaula, 1979; Senshu et al., 2002). If the growth rate is very high in com-

61 parison to surface cooling, this heating can ultimately cause partial or complete

62 melting of the chondritic material (Yoshino et al., 2003) and lead to extensive 
64 et al., 2009).

metal/silicate separation (Tonks and Melosh, 1992; Senshu et al., 2002; Monteux

Hf/W chronology suggests that core formation happened during the first 1030 million years of Mars' history (Lee and Halliday, 1997; Nimmo and Kleine, 2007). Such a rapid process involves extensive melting potentially enhanced by radiogenic heating as a result of the decay of short-lived radionuclides (Yoshino et al., 2003), impact heating (Tonks and Melosh, 1992; Senshu et al., 2002; Monteux et al., 2009) and gravitational energy conversion during metal/silicate separation (Stevenson, 1989; Ricard et al., 2009). Metal/silicate separation can occur via a wide range of phenomena such as percolation (Shannon and Agee, 1996), the sedimentation of metallic rain through a magma ocean (Rubie et al., 2003; Höink et al., 2005) or a large diapir sinking through a solid mantle after an impact (Tonks and Melosh, 1992; Monteux et al., 2009). Whatever the mechanism, Mars' internal structure characterized by a $\sim 1700 \mathrm{~km}$ diameter Fe-core was mostly established within $\sim 10$ Myr of the planet's formation (Yoder et al., 2003) (cf Fig.1a).

The gravitational heat released during martian core formation was partitioned between the planet's core and mantle. The fraction of gravitational heat taken up by the metal or the silicate fraction depends strongly on the rheology of the planet and on the segregation mechanisms (Samuel and Tackley, 2008; Monteux et al., 2009; Ke and Solomatov, 2009). The combined processes leading to core formation yield a wide range of possible early thermal states. In particular, the core could initially have had a temperature close to the deep mantle temperature if thermal equilibration was efficient or it could have been hotter than the mantle if the energy of core formation was mainly retained within the iron. In the latter case, the transition between the hot core and the relatively cooler mantle would occur within a thermal boundary layer with a thickness, $\delta$, 
inversely proportional to the efficiency of core cooling by convection (Fig.1a)

\subsection{Impactor interior}

Assuming that both the impactor and the impacted body had chondritic compositions, their volumetric metal fractions $f_{0}$ should be close (we consider that the impactor has the same metal content as Mars and we use $f_{0}=12.5 \%$ (Stevenson, 2001)). Hence, for 200-1300 km diameter impactors, an additional volume of core material with a radius between $100 \mathrm{~km}$ and $700 \mathrm{~km}$ merges with the preexisting core (Fig.1). The interior temperature of the impactor is unconstrained. However, as the impactor is smaller in radius than Mars, it was probably cooler, on average than the martian interior.

\section{Thermo-chemical state after a mega-impact}

\subsection{Mantle heating and melting}

Although part of the kinetic energy of the impactor is dissipated as a result of the irreversible work done by shock waves to damage crustal rocks, another part of this energy is dissipated thermally within a spherical region (the isobaric core) with a volume $V_{i c}$ that is typically taken to be 3 times larger than the volume of the impactor (O'Keefe and Ahrens, 1977; Croft, 1982; Pierazzo et al., 1997). In this region the temperature increases uniformly from $T_{0}$ to $T_{0}+\Delta T_{0}$, where $T_{0}$ is the temperature of the impacted mantle before the impact and $\Delta T_{0}$ is the temperature increase due to impact heating and melting. Assuming that part of this thermal energy is also consumed to melt the isobaric core and the impactor's iron core with volume $V_{F e}=f_{0} V_{i c} / 3$, this temperature increase is (Monteux et al., 2011):

$$
\Delta T_{0}=\frac{4 / 9 \pi \gamma \rho_{0}^{2} G R^{2}-L_{S i} \rho_{S i}-\frac{f_{0}}{3} L_{F e} \rho_{F e}}{h_{m} \rho_{S i} C_{p, S i}},
$$


Here, $G$ is the gravitational constant, $\rho_{S i}, L_{S i}$ and $\mathrm{C}_{\mathrm{p}, \mathrm{Si}}$ are the density, latent heat and specific heat of the silicate material, $R$ is the radius of the impacted planet and $\gamma$ is the fraction of the kinetic energy of the impactor ultimately dissipated to heat up the mantle (O'Keefe and Ahrens, 1977; Monteux et al., 2007). $L_{F e}$ and $\rho_{F e}$ are the density and latent heat of the impactor's core. As $f_{0}$ is $\mathrm{O}\left(10^{-1}\right)$ then the contribution to $\Delta T_{0}$ from the third term in Eq.1 is negligible and we can simplify Eq.1 to:

$$
\Delta T_{0}=\frac{4 / 9 \pi \gamma \rho_{0}^{2} G R^{2}-L_{S i} \rho_{S i}}{h_{m} \rho_{S i} C_{p, S i}},
$$

The effect of the energy lost as a result of the irreversible work done by shock waves outside the isobaric core is accounted for in the geometric parameter $h_{m}$, which is the volume of rock damaged and heated inside and outside the isobaric core normalized to the volume of the isobaric core itself (see Monteux et al. (2011) for details). From empirical fits to the fall off in pressure with distance from the edge of the isobaric core $h_{m} \approx 2-3$ (Senshu et al., 2002). Using the set of parameters in Table 1 and making the conservative hypothesis that the martian mantle is solid before the impact (i.e. its temperature is just below the melting temperature of the mantle silicates), $\Delta T_{0} \sim 400 \mathrm{~K}$.

Outside the isobaric core, the shock pressure and the excess temperature decrease rapidly with distance $r$ from the isobaric core as approximately $\Delta T_{0}\left(R_{i c} / r\right)^{m}$ (Senshu et al., 2002; Monteux et al., 2009). Hence, the volume effectively heated and damaged after the impact is larger than the isobaric core (see Fig.2). Consequently, when $R_{i m p}>500 \mathrm{~km}$, impact heating occurs within the planetary core. Because the shockwave velocity is lower in liquid than in solid material, the dissipation rate and the impact heating will be weaker in the liquid core than in the surrounding silicate mantle (Arkani-Hamed and Olson, 2010). For simplicity, we will assume that there is no difference in impact heating between 
the martian core and mantle. Hence, for large impactors, our calculations overestimate the direct impact heating within the martian core.

\subsection{The fate of the impactor's material}

During the mega-impact, a significant fraction of the impactor's mantle is ejected far from the impact site and eventually forms a disk of debris. The rest of the impactor's mantle can eventually be incorporated into the mantle of the impacted planet. In contrast, most of the impactor's core is retained within the impacted planet (Canup, 2004) (cf Fig.1b-c), and in our models we assume that $100 \%$ of the impactor's core is retained within the martian mantle after the giant impact. Once incorporated in the impacted planet, the impactor's core can overcome shearing and thinning processes (especially if the impact is oblique) as well as turbulent motions in a magma ocean that can break up large volumes of liquid metal into droplets (Rubie et al., 2003; Deguen et al., 2011; Samuel, 2012). However, the impactor's core may merge with the core of the impacted planet, without emulsification on a droplet lengthscale, if the impactor's core is larger than the thickness of the magma ocean and if the impact is nearly vertical (Rubie et al., 2003; Reese et al., 2010; Dahl and Stevenson, 2010). As mentioned earlier, we consider the pre-impact mantle to be solid, and we also take the impact trajectory to be nearly vertical. Thus, we hypothesize that the impactor's core retains its spherical shape after the impact and that its centre is buried within the martian mantle.

\section{Dynamic models of diapir sinking}

Once buried below the surface, the dense metallic phase from the impactor's core sinks towards the centre of the impacted planet (Fig.1). The large devia- 
toric stress generated by the metallic diapir can lead to a non linear rheology (Samuel and Tackley, 2008), elasto-plastic deformation (Gerya and Yuen, 2007) or even to fracturating processes (Davies, 1982). These produce dissipation in the mantle surrounding the sinking core (Turcotte and Emerman, 1983; Stevenson, 2003). If the iron/silicate viscosity contrast is small, viscous dissipation (i.e. heating) occurs in both the silicate mantle and the sinking core. Alternatively, if the iron/silicate viscosity contrast is large, the viscous dissipation is concentrated in the silicate mantle, but heat can then diffuse into the sinking iron core (Samuel and Tackley, 2008; Monteux et al., 2009).

\subsection{Physical model: Descent of a single diapir}

To investigate the dynamics of the metallic diapir, we adapt the numerical finite volume model in spherical axisymmetric geometry of Monteux et al. (2009). We assume that the mantle is deforming in a diffusion creep limit. The conservation of energy applied to a planet of radius $R$ leads to

$$
\frac{D T}{D t}=\frac{\nabla^{2} T}{R a_{\chi}}+D i\left(\frac{\eta}{\eta_{0}} \Gamma \Omega-v_{r}\left(T+\frac{T_{0}}{\Delta T_{0}}\right) r\right)
$$

where $T, t$ and $r$ are dimensionless temperature, time and radius. $v_{r}$ is the dimensionless radial velocity. $R a_{\chi}=\frac{\Delta \rho_{0} g_{0} R^{3}}{\kappa \eta_{0}}$ is the compositional Rayleigh number and $D i=\frac{\alpha \rho_{0} g_{0} R}{\overline{\rho_{0} C_{p}}}$ is the dissipation number (with $\kappa$ the heat diffusivity and $\overline{\rho_{0} C_{p}}$ the average specific heat of the impacted body). $\Delta \rho_{0}$ is the density difference between metal and silicates. Following Monteux et al. (2009), we introduce the buoyancy ratio $\Gamma=\Delta \rho_{0} /\left(\rho_{0} \alpha \Delta T_{0}\right)$ where $\rho_{0}$ and $\alpha$ are the mean density and thermal expansion of the impacted material (Tab.1). Gravity depends on radius $r$. Within the impacted planet mantle, we consider that gravity is constant $g_{0}$ with $g_{0}=\frac{4}{3} G \pi \rho_{0} R$. Within the core, gravity is a linear function of the radius and $g(r)=g_{0} \frac{r}{R_{c}}$. $\Omega$ is the dimensionless dissipation function and 
expresses the conversion of potential energy into heat :

$$
\Omega=2 \underline{\varepsilon}: \underline{\varepsilon} .
$$

where $\underline{\varepsilon}$ is the dimensionless shear strain rate tensor. Prior to impact, we assume a homogenous temperature $T_{0}$ in the martian mantle. The viscosity is $\eta=\eta_{0} \lambda_{0}^{T}$ where $\lambda_{0}$ is the viscosity ratio (less than 1 ) between the hot impacted material and cold surrounding material far from the impact site, $\eta_{0}$, at the start of the experiment (Ratcliff et al., 1997; Ziethe and Spohn, 2007).

The other dimensionless governing equations are continuity

$$
\boldsymbol{\nabla} \cdot \mathbf{v}=0
$$

and momentum conservation, assuming infinite Prandtl number

$$
-\nabla P+\nabla \cdot\left(\frac{\eta}{\eta_{0}}\left[\nabla \mathbf{v}+[\boldsymbol{\nabla} \mathbf{v}]^{T}\right]\right)+\left(\frac{T}{\Gamma}-f\right) r \mathbf{e}_{\mathbf{r}}=0
$$

where $\mathbf{v}$ and $P$ are the non-dimensional velocity and pressure and $\mathbf{e}_{\mathbf{r}}$ is the radial unit vector. The buoyancy force that drives the flow of the diapir towards the centre of the protoplanet increases with the metallic volume fraction $f$ that varies between 1 (pure metal) and 0 (pure silicates). The metal volume fraction $f$ is then simply advected by the flow :

$$
\frac{D f}{D t}=0
$$

\subsection{Numerical model}

We implement a finite volume numerical model to solve Eq.3, Eq.5, Eq.6 and Eq.7 in axisymmetric spherical geometry. We use a stream function formulation for the equations of motion with a direct implicit inversion method (Schubert 
et al., 2001). Eq.3 and Eq.7 are solved by an Alternating Direction Implicit (ADI) scheme (Peaceman and Rachford, 1955; Douglas, 1955). The stream function, temperature and compositional fields are described by a second-order approximation in space. To limit numerical diffusion when solving the transport equations, especially for the compositional field, we use a Total Variation Diminishing Superbee scheme (Roe, 1986; Laney, 1998) implemented in an implicit way (Srámek et al., 2010) which enables a high resolution of pure advective fields. We use at least $200 \times 400$ grid points. Velocity boundary conditions are free-slip at the surface and along the symmetry axis. Thermal boundary conditions are isothermal at the surface and insulating along the symmetry axis.

\subsection{Analytical model of the core merging process}

Buoyancy-driven interactions between two deformable viscous drops has been widely studied both experimentally and numerically (Davis et al., 1989; Yiantsios and Davis, 1990; Manga and Stone, 1993). Building on this body of work, we characterize the interaction between the preexisting core and the sinking diapir by monitoring the gap distance, $h$, between the surfaces of the impacting core and the planetary core along the axis of symmetry (Fig.1c-d).

We compare the results from our numerical models with analytical predictions. We consider that the initial time $t_{0}$ is the time at which the impact occurs.

During sinking, a diapir will deform the surrounding mantle material, driving a circulation with a spatial extent that depends on the diameter of the diapir and on the rheology of the mantle. When the diapir is small and far from the core-mantle boundary, the presence of the preexisting core has no effect on the diapir motion and the diapir sinks with a classical Rybczynski-Hadamard velocity V (Hadamard, 1911; Rybczynski, 1911) (Fig.3, left)

$$
V=-\frac{d h}{d t}=\frac{a_{0} \Delta \rho_{0} g_{0} R_{F e}^{2}}{\lambda \eta_{0}} .
$$



251 right).

where $h_{0}$ is the initial gap thickness and $t_{s}$ the characteristic time given by

$$
t_{s}=\frac{R_{F e}}{V}=\frac{\lambda \eta_{0}}{a_{0} \Delta \rho_{0} g_{0} R_{F e}}
$$

The Rybczynski-Hadamard velocity gives the terminal velocity of a spherical viscous drop sinking through an infinite viscous fluid. Hence it differs from the Stokes velocity which is the terminal velocity of a solid sphere sinking through an infinite viscous fluid. In our models, the mechanical boundary conditions are applied at a finite distance: the planetary surface and the core-mantle boundary, CMB. Thus, the Rybczynski-Hadamard velocity is only an approximation (Honda et al., 1993; Samuel and Tackley, 2008) and we consider in our models that the time during which this regime occurs is negligible.

When the viscosity of the preexisting planetary core is comparable to or less than that of the mantle, then the planetary core offers little resistance to the radial flow generated by the sinking core. Hence, the CMB acts as a mobile interface. When $h(t) \sim R_{F e}$ and as the diapir approaches the planet's core, the merging core slows down and the planetary core starts to deform while the sinking diapir is weakly deformed (Fig.3, middle). As the distance between the diapir and the CMB decreases, the normal stress difference becomes more localized (Lee and Leal, 1982). When the diapir is at a distance $h(t)<<R_{F e}$ a late lubrication regime is established in the thin gap between the two cores (Fig.3, 
267 by

Integration of Eq.11 leads to

$$
h(t)=h_{0}\left(1-\frac{t}{t_{c}}\right)^{2}
$$

and

$$
t_{c}=\frac{2 h_{0}^{1 / 2} \lambda \eta_{0}}{a_{1} \Delta \rho_{0} g_{0} R_{F e}^{3 / 2}}
$$

271

272 At "late times" (where $h<R_{F e}$ and $t>t_{c}$ ) (see Fig.3, right), the rate at which

$$
V=-\frac{d h}{d t}=\frac{a_{1} \Delta \rho_{0} g_{0} R_{F e}^{3 / 2} h^{1 / 2}}{\lambda \eta_{0}}
$$


the gap thins out as a function of time depends whether the CMB act as a rigid boundary or a mobile interface. According to Jones and Wilson (1978) and Yiantsios and Davis (1990), for two free boundaries the gap thickness should scale with:

$$
h(t)=a_{2} t^{-1 / 3}
$$

where $a_{2}$ is a relative deformation scale $\left(a_{2} \propto B o R_{F e} / h_{0}\right)$ that can be determined from our numerical models and $B o=\Delta \rho_{0} g_{0} R_{F e}^{2} / \sigma$ is the Bond number which is the dimensionless ratio of the buoyancy forces to the surface tension $\sigma$ (Yiantsios and Davis, 1990). In our models, Bo is theoretically infinite since surface tension forces are neglected but has a numerical finite value that can be determined fitting our numerical results with theoretical predictions from Eq.14.

\subsection{Numerical results}

\subsubsection{Initial setup}

We use the numerical model described earlier to characterize the dynamics of core merging after a giant impact on a Mars size body. In this section, the martian core is homogeneously hot with $T=T_{\text {core }}=2000 \mathrm{~K}$ (Roberts and Arkani-Hamed, 2012) (i.e. $\delta=0$ ). The initial thermo-chemical conditions are shown schematically in Fig.2 in which the hatched material represent the metallic phase from the impactor. The initial temperature is represented with a grey scale.

\subsubsection{Evolution of the gap thickness}

Figure 4 represents the thermal and chemical evolution after a $600 \mathrm{~km}$ impact (i.e. $R_{F e}=300 \mathrm{~km}$ ) on a differentiated Mars with homogeneous viscosity $\left(\eta_{0}=10^{22}\right.$ Pa.s). To simplify our study, we fixed the initial gap thickness $h_{0}$ 
(i.e. the depth at which the impactor's core is buried after impact) to a value that does not vary with $R_{i m p}$. We choose $h_{0}=580 \mathrm{~km}$ so that the impactor's core is initially always within the isobaric core for the whole range of impactor radii studied here. The four rows correspond to real time snapshots at $0,0.88$, 2.2, 82.4 Myr respectively. As illustrated in Figure 4, the core from the impactor rapidly sinks toward the pre-existing core. During the sinking, heating occurs at the interface between the diapir and the surrounding mantle (second line, left). Simultaneously, deformation of the pre-existing core accommodates the merging (second line, right). At the end of the chemical merging, the impactor's core is significantly hotter than it was initially (third line). If the end of the merging occurs rapidly (within $6 \mathrm{Myrs}$ ), the thermal readjustement needs a longer time and the extra-heat from impact and viscous dissipation is then removed by a large thermal plume that starts later from the CMB (fourth line).

We monitored the gap thickness $h$ between the front of the merging core and the $\mathrm{CMB}$ as a function of time for different sizes of impact $R_{i m p}$ (equivalent to different sizes of merging core, $R_{F e}$, with $\left.R_{F e}=R_{i m p} / 2\right)$. We used $R_{i m p}$ ranging from 200 to $800 \mathrm{~km}$. Fig.5 illustrates the temporal evolution of the gap thickness (black solid line) for the case illustrated in Fig.4 $\left(R_{i m p}=600\right.$ $\mathrm{km}, R_{F e}=300 \mathrm{~km}, \eta_{0}=10^{22}$ Pa.s and $\left.h_{0}=580 \mathrm{~km}\right)$. We compare the analytical models and the numerical models for both the short early time regime (red dashed line, Eq.12) and the long late time regime (green dashed line Eq.14).

From our numerical results, we obtain the characteristic coalescence times $t_{c}$ and the numerical value for $a_{2}$ for the range of $R_{i m p}$ used in this study. As predicted from Eq.13, our numerical values for $t_{c}$ decrease in proportion to $R_{F e}^{-3 / 2}$ (red circles, Fig. 6). We fit the numerical $t_{c}$ values with the analytical 
prediction (black dashed line, Fig. 6) from Eq.13 and obtained $a_{1}=1 / 9$

4.4.3. Influence of the temperature-dependance of the mantle viscosity on the core merging timescale

The viscosity contrast between the sinking diapir and its environment is a key parameter in understanding the core merging dynamics. As the impactor's core is sinking, shear heating occurs at the interface of the diapir (Samuel et al., 2010) and both the temperature of the diapir and the surrounding mantle increase. Depending on the rheological contrast between the metallic diapir and the mantle, the mean temperature increase within the sinking diapir can reach $\approx 2 \Delta T_{0}$ (Monteux et al., 2009). This thermal modification can influence the sinking dynamics especially if the rheology is temperature dependant. Moreover, as illustrated in Fig.3, viscous dissipation may also occur in the planetary core as a result of the shear flow produced at the CMB during the late stages of core merging especially in the non realistic case where the planetary core and its mantle have the same viscosity (i.e., the core viscosity is way too large compared to the real problem). However, this heating process is negligible compared to the temperature increase induced by the merging of a hot diapir in the preexisting core.

We introduced a temperature-dependent viscosity in our core-merging models. We chose the same initial thermal state as previously used with a core hotter than the surrounding mantle $\left(T_{\text {core }}>T_{0}\right)$. As the temperature of the impacted mantle and of the impactor's core are initially equal, the impactor's core initially starts to sink in a medium with an homogenous temperature. Hence, our results illustrate the effect of viscous heating of the surrounding material on the diapir dynamics. In our models, the viscosity varies with $\eta=\eta_{0} \lambda_{0}^{T}$ (with $T$ the normalized temperature), hence the viscosity within the impacted mantle will be 
$\approx \eta_{0} \lambda_{0}$ while the mean viscosity of the metallic diapir at the end of the sinking will be $\approx \eta_{0} \lambda_{0}^{2}$ (since its temperature increases by $\sim 2 \Delta T_{0}$ ). Consequently, a decrease of $\lambda_{0}$ by one order in magnitude in our models should decrease the viscosity contrast between the diapir and the surrounding material and the sinking time by one order of magnitude. However, the viscosity contrast between the diapir and the cold mantle far from the impact site will reach approximately two orders of magnitude.

To measure the effect of the rheology on the coalescence time, we plot $h(t)$ in Figure 7 for four different viscosity parameters: $\left(\eta_{0}=10^{22}\right.$ Pa.s, $\left.\lambda_{0}=1\right)$, $\left(\eta_{0}=10^{20}\right.$ Pa.s, $\left.\lambda_{0}=1\right),\left(\eta_{0}=10^{22}\right.$ Pa.s, $\left.\lambda_{0}=0.1\right)$ and $\left(\eta_{0}=10^{22}\right.$ Pa.s, $\left.\lambda_{0}=0.01\right)$ with $R_{F e}=300 \mathrm{~km}\left(R_{i m p}=600 \mathrm{~km}\right)$ and $h_{0}=580 \mathrm{~km}$ (see Figure 7). When the viscosity is homogenous, the preexisting core has an influence on the core merging dynamics and Eq.12 is valid. However, where viscous dissipation heats the sinking diapir and softens the surrounding mantle, core merging is faster and the sinking dynamics is close to a classic Stokes regime with a terminal Rybczynski-Hadamard velocity in agreement with Eq.9. We fit uniform viscosity evolutions for $h(t)$ with Eq.12 and temperature dependent evolutions for $h(t)$ with Eq.9 and obtain the characteristic sinking times listed in Tab.2.

For a uniform viscosity, the decrease of $\eta_{0}$ from $10^{22}$ to $10^{20}$ Pa.s reduces the characteristic sinking time from $1.56 \mathrm{Myr}$ to $15.6 \mathrm{kyr}$. For a temperature dependant viscosity, the characteristic sinking time decreases as $\lambda$ decreases. As predicted from Eq.10 and Eq.13, the relation between the characteristic sinking time and the rheology contrast is almost linear (Table 1). 


\section{Effect on the preexisting dynamo}

We now discuss the consequences of a giant-impact and the subsequent core merging process on the martian dynamo generation. We first list the conditions required for dynamo generation and then measure the influence of the core merging on the efficiency of core cooling for different structures of the thermal boundary layer within the planetary core before the impact.

\subsection{Conditions required for dynamo generation}

As discussed in Monteux et al. (2011), three conditions are required to generate a dynamo on a growing planet:

1. The mean heat flux $q_{C M B}$ out of the core must exceed the adiabatic value $q_{A}$ such that convection can occur (Stevenson et al., 1983). This condition is

$$
q_{C M B}>q_{A}=\frac{k_{F e} \alpha_{F e} g_{c} T_{c}}{C_{p, F e}}
$$

where $k_{F e}, \alpha_{F e}, T_{c}$ and $C_{p, F e}$ are respectively the thermal conductivity, the thermal expansion, the temperature and the heat capacity of the metallic phase and $g_{c}$ is the gravity at the surface of the protocore. The largest uncertainties in the calculation of $q_{A}$ concern $T_{c}$ and $k_{F e}$. Assuming reasonable values, convection in the martian core should start once $q_{C M B}>q_{A}=5-19 \mathrm{~mW} \cdot \mathrm{m}^{-2}$ (Nimmo and Stevenson, 2000) (see Tab.1 for values).

2. The ratio of the rate at which gravitational potential energy is released by convection to the rate of ohmic dissipation, $\Phi$, must exceed a critical value (Buffett, 2002): 


$$
\frac{4 \pi R_{c}^{2} q_{C M B}}{\Phi}>\frac{1}{\epsilon_{T}} .
$$

Here, $\epsilon_{T}$ is the Carnot-style efficiency for thermal convection. Here, we do not consider the effect of chemical convection or the presence of an inner core. Assuming that the characteristic length scale of the flow leading to magnetic field generation is the radius of the protocore, $\Phi$ can be approximated as (Buffett, 2002):

$$
\Phi=\left(\frac{\nu \bar{B}^{2}}{\mu}\right) \frac{4}{3} \pi R_{c}
$$

where $\bar{B}$ is the average strength of the magnetic field within the core, $\nu$ is the magnetic diffusivity and $\mu$ is the magnetic permeability (see Tab.1 for values). In the absence of constraints on $\bar{B}$ for early planets, we assume a current Earth-like value of $2.5 \mathrm{mT}$ (Kuang and Bloxham, 1997) that is independent of the protocore size or the planetary radius. The efficiency of thermal convection is given by (Buffett, 2002)

$$
\epsilon_{T}=\frac{0.8 \pi}{3} \frac{\alpha_{F e} G \rho_{F e} R_{c}^{2}}{C_{p, F e}}\left(1-\frac{q_{A}}{q_{C M B}}\right)
$$

with $\rho_{F e}$ the density of the metallic phase and $G$ the gravitational constant.

Hence Eq.16, Eq.17 and Eq.18 lead to:

$$
q_{C M B}-q_{A}>\frac{\nu \bar{B}^{2} C_{p, F e}}{0.8 \pi G \mu \alpha_{F e} \rho_{F e} R_{c}^{3}} \approx 8 . \times 10^{-2} \mathrm{~mW} \cdot \mathrm{m}^{-2}
$$

3. The structure of the convective motions carrying magnetic field lines must be sufficiently complicated to favor self-sustaining dynamo action. A measure of this complexity is that the magnetic Reynolds number (Christensen 
and Aubert, 2006)

$$
R e_{m}=\frac{U L}{\nu}>R e_{m}^{c r i t}=O\left(10-10^{2}\right)
$$

Here, $L$ and $U$ are the characteristic length and velocity scales for the flow within the protocore and $\nu$ is the magnetic diffusivity of the metal phase. Whereas the natural length scale in the problem is the depth of the convecting iron layer, the choice of an appropriate velocity scale depends on the leading order force balance (Christensen, 2010). As the rotation rate of growing planets is potentially time-dependent and poorly constrained a convenient and reasonable choice is based on a balance between inertial and buoyancy forces and is (Stevenson, 2003):

$$
U \sim\left(\frac{\left(q_{C M B}-q_{A}\right) \alpha_{F e} g_{c} R_{F e}}{\rho_{F e} C_{p, F e}}\right)^{1 / 3}
$$

Taking $L=R_{c}$ and $R e_{m}^{c r i t}=10^{2}$, the combination of Eq.20 and Eq.21 leads to the condition

$$
q_{C M B}-q_{A}>\frac{\rho_{F e} C_{p, F e}}{\alpha_{F e} g_{c} R_{c}}\left(R e_{m}^{c r i t} \frac{\nu}{R_{c}}\right)^{3} \approx 10^{-4} \mathrm{~mW} \cdot \mathrm{m}^{-2}
$$

Among the three criteria above, the first is typically considered a necessary condition for a thermally-driven dynamo (Subadiabatic dynamos are possible if e.g., compositional gradients also drive convection.). However it is not a sufficient condition for dynamo action, as indicated by the other two conditions. To get the two last conditions, the heat flux $q_{C M B}$ at the CMB has to be larger than the adiabatic heat flux $q_{A}$ but the difference required is small (between 8. $\times 10^{-2}$ and $\left.10^{-4} \mathrm{~mW} \cdot \mathrm{m}^{-2}\right)$. Hence we will consider from now that any heat 35 flux larger than $q_{A}$ can potentially lead to a dynamo generation (Nimmo and 
Stevenson, 2000; Williams and Nimmo, 2004).

\subsection{Structure of the thermal boundary layer within the core before the impact}

To understand the thermal influence of the merging process on the thermallydriven dynamo action, we need to have an idea of the structure of the thermal boundary layer on the core side of the core-mantle boundary prior to impact, which depends on the previous accretion and core formation histories (Monteux et al., 2009; Samuel et al., 2010). Two end-member models for the initial interior thermal state of Mars are the isothermal case with the core and the mantle having the same temperature, and a situation where the core is much hotter than the mantle.

In the case of a pre-impact core hotter than the mantle, the thickness $\delta$ of the TBL is governed by the mode of heat loss to the mantle. Whereas strong cooling to the mantle leads to thin thermal boundary layers and vigorous convection, weak cooling leads to relatively more sluggish flow or no convection at all. To simplify this problem we consider three pre-impact core thermal states: an isothermal core and mantle, and two models where the core is hotter than the mantle with $\delta=0.1 R_{F e}$ (strong core cooling) and $\delta=0.4 R_{F e}$ (weak core cooling). In the last two models the temperature decreases linearly in the thermal boundary layer from the planetary core where we impose $T=T_{\text {core }}$ to the CMB where $T=T_{0}$.

\subsection{Evolution of the CMB heat flow after impact: Numerical results.}

Following a mega-impact extensive mantle heating causes the average core heat flow to decline or reverse direction. This phenomenon has been inferred to explain the cessation of the martian dynamo (Roberts et al., 2009; Watters 
et al., 2009). For large impactors, the resulting core heating can lead to stratification within the core and subsequent dynamo cessation (Arkani-Hamed and Olson, 2010). However lower mantle heating from the impact can drive and focus the formation of mantle plumes, which in turn increases core heat loss and can enhance the strength and longevity of a dynamo (Reese et al., 2010).

Depending on the pre-impact core formation and accretionary heating histories, as well as the energetics of post-impact sinking discussed in section 3, a wide range of early martian thermal states are plausible. We monitor $q_{C M B}$ for different size of impactors (i.e. merging core radii), viscosity contrasts and efficiencies of core cooling (i.e. thermal boundary layer thickness). Assuming perfect thermal mixing of the pre- and post-impact cores, Fig. 8 shows that core merging leads to a significant increase in the mean heat flux $q_{C M B}$. Our results show that a heat flux sufficiently large to drive a dynamo is favored for large impacts, low viscosities and for strongly-cooled pre-impact cores. In addition, dynamo longevity is enhanced for low core viscosities.

\subsection{Consequences for the dynamo generation}

Among the recent models that correlate giant-impact and martian dynamo activity (Roberts et al., 2009; Watters et al., 2009; Arkani-Hamed and Olson, 2010; Reese et al., 2010), only the last one considers the thermal consequences of the core merging and assumes the formation of a hot layer at the CMB. However, none of these models consider the viscous dissipation during the core merging and the subsequent thermal perturbation at the CMB. Even if our model does consider this effect, the results from the previous section have to be considered carefully. The dynamo generation process is not a straightforward consequence of the core merging process and some assumptions on the thermal mixing within the martian core are needed to correlate core merging and dy- 
namo histories. Indeed, if thermal mixing is not perfect and the merging core forms a hot layer at the CMB this thermal stratification may kill a thermally driven dynamo (Arkani-Hamed and Olson, 2010). The heat flux at the CMB might be greater than the critical value for a dynamo but the heat flux into the core might be large enough to make it go subadiabatic. However, if the hot layer mixes efficiently (Fig.1f), our results may give a picture of the dynamo generation after a giant impact.

Assuming perfect thermal mixing, our results show that dynamo initiation might be favoured by large impacts, low viscosity of the preexisting core and strong core cooling (i.e. thin thermal boundary layer). Assuming that dynamo longevity depends on how long it takes to remove the added thermal anomaly, post-impact dynamo can last several hundred Myrs. Whether or not the new core material will mix as it spreads is not certain. The answer depends on the density contrast between the merging core and the preexisting core and in particular how hot is the merging core. The mixing also depends on the strength of the preexisting core convection. Whether ambient convective motions plus the shear related to the spread of the hot current cause mixing has to be calculated and depends on the balance between driving inertia and stabilizing buoyancy forces.

In our models, the mean heat flux $q_{C M B}$ is overestimated because all the hot merging material stays at the CMB. However, we treat the core and mantle as having the same viscosity so the spreading of the new core material is in the wrong dynamical limit. Considering the effect of core merging on the core dynamics in a more realistic way (i.e. with finite Reynolds number) is currently beyond the scope of this paper and will be developed in a separated study in 
which our results will constrain the initial thermal conditions within the core. Mixing will be enhanced at very high Reynolds number, depending on the density difference between the intruded and pre-exisiting cores (see e.g. Linden $(1977,1979))$.

On the other hand, our results are also quite conservative since we assume that the giant impact occurs with the martian escape velocity. When the two planetary embryos have collided near the end of accretion, the impact velocity was probably higher than that of the planetesimals during the accretion period. Considering larger impact velocities can severely increase the post-impact temperature in the mantle and in the merging core before the sinking from Eq.1 and Eq.2. Hence, we give only a lower bond of the temperature increase in the core after the diapir merging.

\section{Conclusions}

Giant impacts may have played an important role in the thermal and magnetic evolutions of terrestrial planets. The giant impact forming the martian dichotomy could have supplied a significant volume of iron that has merged with the preexisting core. The size of the impact and the rheology strongly influenced the dynamics of core merging which probably occurred in less than a million years. During the sinking, the mantle and the merging core have overcome viscous heating which have modified the thermal state at the core mantle boundary. Hence, depending on the mixing efficiency in the core, the heat supply might have enhanced a dynamo activity.

Our models better constrain the dynamics of the sinking of a large metallic diapir within a silicate mantle and especially the influence of the deformation of 
the preexisting core on the merging process. We obtain characteristic timescales of the coalescence time between the two cores as a function of the viscosity of the mantle and the size of the sinking diapir. Our results are in agreement with theoretical and experimental models of buoyancy-driven motions sinking toward a deformable surface. We also show that the thermal consequences of the merging process can influence the generation of a martian dynamo. Assuming perfect thermal mixing, the dynamo generation might be favoured by large impacts, low viscosity of the preexisting core and strong preexisting core cooling. This dynamo can eventually last several hundred million years depending on the mixing process within the core. Our results also illustrate the importance of better constraining the thermal states of the cores, the size of the biggest diapir that can remain consistent after a giant impact and the depth at which the impactor's core is buried before experiencing viscous deformation and core merging process.

The heating induced by the impact within the mantle and the viscous dissipation during diapir sinking have enhanced a thermal anomaly within the martian mantle and, hence, a dichotomy of the core cooling. Indeed, the core heat flux at the CMB below the impact site was probably different than the heat flux at the opposite side of the core. This mantle heterogeneity might affect the dynamo and the paleomagnetic field recorded at the surface (Stanley et al., 2008). Moreover, recent results show that the efficiency of a mantle heterogeneity centred at the geographical pole in producing a south-north dichotomy is much higher than that of an heterogeneity centred at the equator in producing an east-west dichotomy (Amit et al., 2011). Hence, our models may have interesting implications in the understanding of the structure of the past martian dynamo.

\section{Acknowledgements}


572

${ }_{573}$ Acuña, M., et al. (1999), Global distribution of crustal magnetization discovered

J. Monteux is funded by Agence Nationale de la Recherche (Accretis decision $\mathrm{n}^{\circ}$ ANR-10-PDOC-001-01). M. Jellinek and C.L. Johnson acknowledge support from the Natural Science and Engineering Research Council of Canada.

\section{References}

575

576

by the mars global surveyor MAG/ER experiment, Science, 284, 790-793.

Agee, C. B. (1997), Melting temperatures of the Allende meteorite: implications for a Hadean magma ocean, Phys. Earth Planet. Int., 100, 41-47.

Amit, H., U. Christensen, and B. Langlais (2011), The influence of degree-1 mantle heterogeneity on the past dynamo of Mars, Phys. Earth Planet. Int., p. in press.

Andrews-Hanna, J. C., M. T. Zuber, and W. B. Banerdt (2008), The Borealis basin and the origin of the martian crustal dichotomy, Nature, 453, 12121215, doi:10.1038/nature07011.

Arkani-Hamed, J., and P. Olson (2010), Giant impact stratification of the Martian core, Geophys. Res. Lett., 370, L02,201, doi:10.1029/2009GL041417.

Benz, W., W. L. Slattery, and A. G. W. Cameron (1988), Collisional stripping of Mercury's mantle, Icarus, 74, 516-528, doi:10.1016/0019-1035(88)90118-2.

Buffett, B. A. (2002), Estimates of heat flow in the deep mantle based on the power requirements for the geodynamo, Geophys. Res. Lett., 29(12), 1-4.

Canup, R. M. (2004), Simulations of a late lunar-forming impact, Icarus, 168, 433-456, doi:10.1016/j.icarus.2003.09.028.

Christensen, U. R. (2010), Dynamo Scaling Laws and Applications to the Planets, Space Sci. Rev., 152, 565-590. 
Christensen, U. R., and J. Aubert (2006), Scaling properties of convectiondriven dynamos in rotating spherical shells and application to planetary magnetic fields, Geophys. J. Int., 166, 97-114.

Croft, S. K. (1982), A first-order estimate of shock heating and vaporization in oceanic impacts, vol. 190, 143-152 pp., Geological Implications of Impacts of Large Asteroids and Comets on Earth, edited by T.L. Silver and P.H. Schultz,Spec. Pap. Geol. Soc. Am.

Dahl, T. W., and D. J. Stevenson (2010), Turbulent mixing of metal and silicate during planet accretion and interpretation of the Hf-W chronometer, Earth and Planetary Science Letters, 295, 177-186, doi:10.1016/j.epsl.2010.03.038.

Davies, G. F. (1982), Ultimate strength of solids and formation of planetary cores, Geophys. Res. Lett., 9, 1267-1270.

Davis, R. H., J. A. Schonberg, and J. M. Rallison (1989), The lubrication force between two viscous drops, Physics of Fluids, 1, 77-81, doi:10.1063/1.857525.

Deguen, R., P. Olson, and P. Cardin (2011), Experiments on turbulent metalsilicate mixing in a magma ocean, Earth and Planetary Science Letters, 310, 303-313, doi:10.1016/j.epsl.2011.08.041.

Douglas, J. (1955), On the numerical integration of $\frac{\partial^{2} u}{\partial x^{2}}+\frac{\partial^{2} u}{\partial y^{2}}=\frac{\partial u}{\partial t}$ by implicit methods, J. Soc. Ind. Appl. Math., 3, 42-65, doi:10.1137/0103004.

Elkins-Tanton, L. T., E. M. Parmentier, and P. C. Hess (2003), Magma ocean fractional crystallization and cumulate overturn in terrestrial planets: Implications for Mars, Meteoritics and Planetary Science, 38, 1753-1771.

Frey, H. V. (2006), Impact constraints on the age and origin of the lowlands of Mars, Geophys. Res. Lett., 33, 8-+, doi:10.1029/2005GL024484. 
Gerya, T. V., and D. A. Yuen (2007), Robust characteristics method for modelling multiphase visco-elastic thermo-mechanical problems, Phys. Earth Planet. Int., 163, 83-105.

Hadamard, J. (1911), Mouvement permanent lent d'une sphère liquide et visqueuse dans un liquide visqueux, C. R. Acad. Sci., 152, 1735-1738.

Hartmann, W. K., and D. R. Davis (1975), Satellite-sized planetesimals and lunar origin, Icarus, 24, 504-514.

Höink, T., J. Schmalzl, and U. Hansen (2005), Formation of compositional structures by sedimentation in vigorous convection, Phys. Earth Planet. Int., $153,11-20$.

Honda, R., H. Mizutani, and T. Yamamoto (1993), Numerical simulation of Earth's core formation., J. Geophys. Res., 98, 2075-2090.

Hood, L. L., N. C. Richmond, E. Pierazzo, and P. Rochette (2003), Distribution of crustal magnetic fields on Mars: Shock effects of basin-forming impacts, Geophys. Res. Lett., 30(6), 1-4.

Johnson, C. L., and R. J. Phillips (2005), Evolution of the Tharsis region of Mars: insights from magnetic field observations, Earth and Planet. Sci. Lett., 230, 241-254.

Jones, A. F., and S. D. R. Wilson (1978), The film drainage problem in droplet coalescence, Journal of Fluid Mechanics, 87, 263-288, doi: $10.1017 /$ S0022112078001585.

Kaula, W. M. (1979), Thermal evolution of earth and moon growing by planetesimal impacts, J. Geophys. Res., 84, 999-1008.

Ke, Y., and V. S. Solomatov (2009), Coupled core-mantle thermal evolution of early Mars, Journal of Geophysical Research (Planets), 114(13), 1-12. 
Kuang, W., and J. Bloxham (1997), An Earth-like numerical dynamo model, Nature, 389, 371-374.

Laney, C. B. (1998), Computational gasdynamics, Cambridge University Press, Cambridge.

Lee, D. C., and A. N. Halliday (1997), Core formation on Mars and differentiated asteroids, nat, 388, 854-857.

Lee, S. H., and L. G. Leal (1982), The motion of a sphere in the presence of a deformable interface : Ii. a numerical study of the translation of a sphere normal to an interface, Journal of Colloid and Interface Science, 87(1), 81 106.

Lillis, R. J., H. V. Frey, and M. Manga (2008a), Rapid decrease in Martian crustal magnetization in the Noachian era: Implications for the dynamo and climate of early Mars, Geoph. Res. Lett., 35, 14,203-+.

Lillis, R. J., H. V. Frey, M. Manga, D. L. Mitchell, R. P. Lin, M. H. Acuña, and S. W. Bougher (2008b), An improved crustal magnetic field map of Mars from electron reflectometry: Highland volcano magmatic history and the end of the martian dynamo, Icarus, 194, 575-596.

Linden, P. F. (1977), The flow of a stratified fluid in a rotating annulus, Journal of Fluid Mechanics, 79, 435-447, doi:10.1017/S0022112077000251.

Linden, P. F. (1979), Mixing in stratified fluids, Geophysical and Astrophysical Fluid Dynamics, 13, 3-23, doi:10.1080/03091927908243758.

Manga, M., and H. A. Stone (1993), Buoyancy-driven interactions between two deformable viscous drops, Journal of Fluid Mechanics, 256, 647-683, doi: $10.1017 / \mathrm{S} 0022112093002915$. 
Marinova, M. M., O. Aharonson, and E. Asphaug (2008), Mega-impact formation of the Mars hemispheric dichotomy, Nature, 453, 1216-1219, doi: 10.1038/nature07070.

Marinova, M. M., O. Aharonson, and E. Asphaug (2011), Geophysical consequences of planetary-scale impacts into a Mars-like planet, Icarus, 211, 960-985, doi:10.1016/j.icarus.2010.10.032.

Monteux, J., N. Coltice, F. Dubuffet, and Y. Ricard (2007), Thermo-mechanical adjustment after impacts during planetary growth, Geophys. Res. Lett., 34, $24,201-24,205$.

Monteux, J., Y. Ricard, N. Coltice, F. Dubuffet, and M. Ulvrova (2009), A model of metal-silicate separation on growing planets, Earth and Planet. Sci. Lett., 287, 353-362.

Monteux, J., A. M. Jellinek, and C. L. Johnson (2011), Why might planets and moons have early dynamos?, Earth and Planetary Science Letters, 310, 349-359, doi:10.1016/j.epsl.2011.08.014.

Nimmo, F., and T. Kleine (2007), How rapidly did Mars accrete? Uncertainties in the $\mathrm{Hf} \mathrm{W}$ timing of core formation, Icarus, 191, 497-504, doi: 10.1016/j.icarus.2007.05.002.

Nimmo, F., and D. J. Stevenson (2000), Influence of early plate tectonics on the thermal evolution and magnetic field of Mars, J. Geophys. Res., 105, $11,969-11,980$.

Nimmo, F., S. D. Hart, D. G. Korycansky, and C. B. Agnor (2008), Implications of an impact origin for the martian hemispheric dichotomy, Nature, 453, 12201223, doi:10.1038/nature07025. 
O'Keefe, J. D., and T. J. Ahrens (1977), Impact-induced energy partitioning, melting, and vaporization on terrestrial planets, in Lun. Planet. Sci. Conf., vol. 8, edited by R. B. Merril, pp. 3357-3374.

Peaceman, D. W., and H. H. Rachford (1955), The numerical solution of parabolic and elliptic differential equations, J. Soc. Ind. Appl. Math., 3, 2841, doi:10.1137/0103003.

Pierazzo, E., A. M. Vickery, and H. J. Melosh (1997), A Reevaluation of Impact Melt Production, Icarus, 12\%, 408-423.

Ratcliff, J. T., P. J. Tackley, G. Schubert, and A. Zebib (1997), Transitions in thermal convection with strongly variable viscosity, Phys. Earth Planet. Int., 102, 201-212.

Reese, C. C., C. P. Orth, and V. S. Solomatov (2010), Impact origin for the Martian crustal dichotomy: Half emptied or half filled?, Journal of Geophysical Research (Planets), 115(14), 5004-+, doi:10.1029/2009JE003506.

Ricard, Y., O. Srámek, and F. Dubuffet (2009), A multi-phase model of runaway core-mantle segregation in planetary embryos, Earth and Planet. Sci. Lett., 284, 144-150.

Roberts, J. H., and J. Arkani-Hamed (2012), Impact-induced mantle dynamics on Mars, Icarus, 218, 278-289, doi:10.1016/j.icarus.2011.11.038.

Roberts, J. H., and S. Zhong (2006), Degree-1 convection in the Martian mantle and the origin of the hemispheric dichotomy, Journal of Geophysical Research (Planets), 111(10), 6013-+, doi:10.1029/2005JE002668.

Roberts, J. H., R. J. Lillis, and M. Manga (2009), Giant impacts on early Mars and the cessation of the Martian dynamo, J. Geophys. Res. (Planets), $114(13), 4009-+$. 
Roe, P. L. (1986), Characteristic-based schemes for the Euler equations, Annual Review of Fluid Mechanics, 18, 337-365.

Rubie, D. C., H. J. Melosh, J. E. Reid, C. Liebske, and K. Righter (2003), Mechanisms of metal-silicate equilibration in the terrestrial magma ocean, Earth and Planet. Sci. Lett., 205, 239-255.

Rybczynski, W. (1911), über die fortschreitende bewegung einer flüssigen kugel in einen medium, Bull. Acad. Sci. Cracovie, 1, 40-46.

Safronov, V. S. (1978), The heating of the Earth during its formation, Icarus, 33, 3-12, doi:10.1016/0019-1035(78)90019-2.

Samuel, H. (2012), A re-evaluation of metal diapir breakup and equilibration in terrestrial magma oceans, Earth and Planetary Science Letters, 313, 105-114, doi:10.1016/j.epsl.2011.11.001.

Samuel, H., and P. J. Tackley (2008), Dynamics of core formation and equilibration by negative diapirism, Geochem. Geophys. Geosyst., 9, 6011-6026.

Samuel, H., P. J. Tackley, and M. Evonuk (2010), Heat partitioning in terrestrial planets during core formation by negative diapirism, Earth and Planetary Science Letters, 290, 13-19.

Schubert, G., D. L. Turcotte, and P. Olson (2001), Mantle convection in the Earth and planets, Cambridge University Press.

Senshu, H., K. Kuramoto, and T. Matsui (2002), Thermal evolution of a growing Mars, J. Geophys. Res., 107, 1-13.

Shannon, M. C., and C. B. Agee (1996), High pressure constraints on percolative core formation, Geophys. Res. Lett., 23, 2717-2720. 
Smith, J. V. (1979), Mineralogy of the Planets: a Voyage in Space and Time, Mineralogical Magazine, 43, 1-89.

Stanley, S., L. Elkins-Tanton, M. T. Zuber, and E. M. Parmentier (2008), Mars' Paleomagnetic Field as the Result of a Single-Hemisphere Dynamo, Science, 321, 1822-, doi:10.1126/science.1161119.

Stevenson, D. J. (1989), Formation and early evolution of the Earth, 818-868 pp., in Mantle convection and plate tectonics, W.R. Peltier, ed.

Stevenson, D. J. (2001), Mars' core and magnetism, Nature, 412, 214-219.

Stevenson, D. J. (2003), Planetary magnetic fields, Earth and Planetary Science Letters, 208, 1-2.

Stevenson, D. J., T. Spohn, and G. Schubert (1983), Magnetism and thermal evolution of the terrestrial planets, Icarus, 54, 466-489.

Tonks, W. B., and H. J. Melosh (1992), Core formation by giant impacts, Icarus, 100, 326-346.

Turcotte, D. L., and S. H. Emerman (1983), Dissipative melting as a mechanism for core formation., Journ. Geophys, Res., 88, 91, doi: 10.1029/JB088iS01p00B91.

Srámek, O., Y. Ricard, and F. Dubuffet (2010), A multiphase model of core formation, Geophysical Journal International, 181, 198-220, doi:10.1111/j.1365246X.2010.04528.x.

Watters, W. A., M. T. Zuber, and B. H. Hager (2009), Thermal perturbations caused by large impacts and consequences for mantle convection, Journal of Geophysical Research (Planets), 114, E02,001, doi:10.1029/2007JE002964. 
761 Wilhelms, D. E., and S. W. Squyres (1984), The martian hemispheric dichotomy 762 may be due to a giant impact, Nature, 309, 138-140, doi:10.1038/309138a0.

763 Williams, J., and F. Nimmo (2004), Thermal evolution of the Martian core:

764 Implications for an early dynamo, Geology, 32.

765 Yiantsios, S. G., and R. H. Davis (1990), On the buoyancy-driven motion of 766 a drop towards a rigid surface or a deformable interface, Journal of Fluid ${ }_{767}$ Mechanics, 217, 547-573, doi:10.1017/S0022112090000842.

${ }_{768}$ Yoder, C. F., A. S. Konopliv, D. N. Yuan, E. M. Standish, and W. M. Folkner 769 (2003), Fluid Core Size of Mars from Detection of the Solar Tide, Science, $770 \quad 300,299-303$, doi:10.1126/science.1079645.

771 Yoshino, T., M. J. Walter, and T. Katsura (2003), Core formation in planetesi772 mals triggered by permeable flow, Nature, 422, 154-157.

773 Ziethe, R., and T. Spohn (2007), Two-dimensional stokes flow around a heated ${ }_{774}$ cylinder: A possible application for diapirs in the mantle, J. Geophys. Res., $775 \quad 112,1-13$. 
Table 1: Typical parameter values for numerical models

\begin{tabular}{lcc}
\hline Mars radius & $R$ & $3400 \mathrm{~km}$ \\
Mars core radius & $R_{c}$ & $1700 \mathrm{~km}$ \\
Impactor radius & $R_{i m p}$ & $200-800 \mathrm{~km}$ \\
Impactor core radius & $R_{F e}$ & $100-400 \mathrm{~km}$ \\
Density difference & $\Delta \rho_{0}$ & $4500 \mathrm{~kg} \mathrm{~m}^{-3}$ \\
Average density & $\rho_{0}$ & $4060 \mathrm{~kg} \mathrm{~m}^{-3}$ \\
Iron density & $\rho_{F e}$ & $8000 \mathrm{~kg} \mathrm{~m}^{-3}$ \\
Silicate density & $\rho_{S i}$ & $3500 \mathrm{~kg} \mathrm{~m}^{-3}$ \\
Mean thermal expansion & $\alpha$ & $4.5 \times 10^{-5} \mathrm{~K}^{-1}$ \\
Iron thermal expansion & $\alpha_{F e}$ & $1.5 \times 10^{-5} \mathrm{~K}^{-1}$ \\
Silicate thermal expansion & $\alpha_{S i}$ & $5 \times 10^{-5} \mathrm{~K}^{-1}$ \\
Iron heat capacity & $C_{p, F e}$ & $800 \mathrm{~J} \mathrm{~K}^{-1} \mathrm{~kg}^{-1}$ \\
Silicate heat capacity & $C_{p, S i}$ & $1000 \mathrm{~J} \mathrm{~K}^{-1} \mathrm{~kg}^{-1}$ \\
Iron latent heat & $L_{F e}$ & $2.7 \times 10^{5} \mathrm{~J} \mathrm{~kg}^{-1}$ \\
Silicates latent heat & $L_{S i}$ & $4 \times 10^{5} \mathrm{~J} \mathrm{~kg}^{-1}$ \\
Pre-impact mantle temperature & $T_{0}$ & $1600 \mathrm{~K}^{-1}$ \\
Heat diffusivity & $\chi$ & $10^{-6} \mathrm{~m}^{2} \mathrm{~s}^{-1}$ \\
Thermal conductivity & $k$ & $4 \mathrm{~W} \mathrm{~m} \mathrm{~K}^{-1}$ \\
Metal content & $f_{0}$ & $12.5 \%$ \\
Reference viscosity & $\eta_{0}$ & $10^{20}-10^{22} \mathrm{~Pa} \mathrm{~s}^{-}$ \\
Impact energy conversion & & \\
coefficient & $\gamma$ & 0.3 \\
Volume effectively heated & & \\
by impact & $h(m)$ & 2.7 \\
Gravitational constant & $\mathrm{G}$ & $6.67 \times 10^{-11} \mathrm{~m}^{3} \mathrm{~kg}^{-1} \mathrm{~s}^{-2}$ \\
Average magnetic field strength & $B$ & $2.5 \mathrm{mT}^{2}$ \\
Magnetic diffusivity & $\nu$ & $2 \mathrm{~m}^{2} \mathrm{~s}^{-1}$ \\
Magnetic permeability & $\mu$ & $4 \pi \times 10^{-7} \mathrm{H} \mathrm{m}{ }^{-1}$ \\
\hline & &
\end{tabular}


Table 2: Sinking time values obtained fitting numerical experiments with theoretical predictions ( Eq.9 and Eq.12) for different values of $\eta_{0}$ and $\lambda$ (with $R_{i m p}=600 \mathrm{~km}, h_{0}=580$ $\mathrm{km})$

\begin{tabular}{ccccc} 
& $\eta_{0}=10^{22}$ Pa.s & $\eta_{0}=10^{20}$ Pa.s & $\eta_{0}=10^{22}$ Pa.s & $\eta_{0}=10^{22}$ Pa.s \\
& $\lambda=1$ & $\lambda=1$ & $\lambda=0.1$ & $\lambda=0.01$ \\
\hline$t$ & $1.56 \mathrm{Myr}$ & $1.56 \times 10^{-2} \mathrm{Myr}$ & $9.5 \times 10^{-2} \mathrm{Myr}$ & $9.5 \times 10^{-3} \mathrm{Myr}$ \\
\hline
\end{tabular}




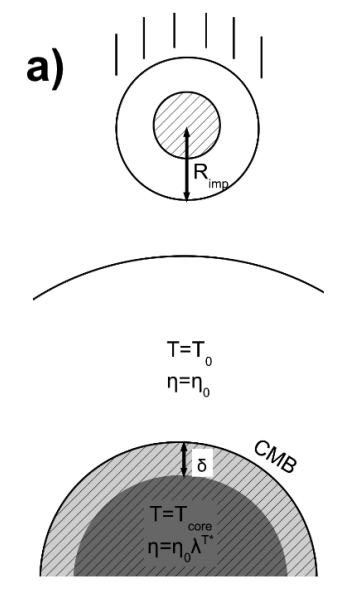

b)

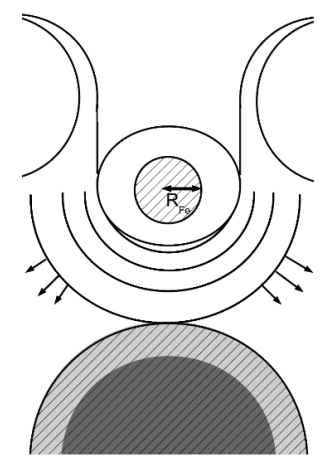

e)

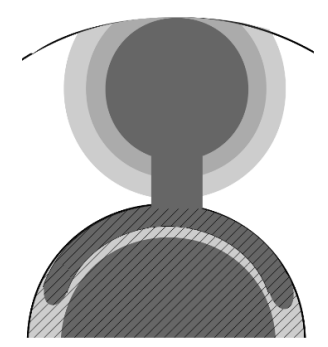

c)

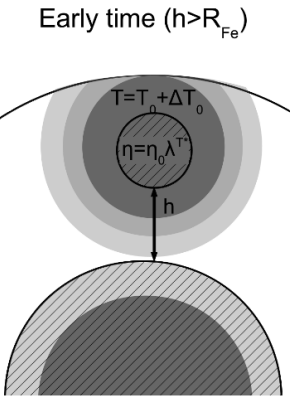

f)

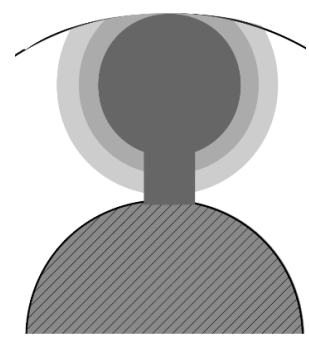

Figure 1: Schematic representation of the thermo-chemical evolution following a mega-impact on a Mars-size body. After the impact (a) and the subsequent shockwave propagation (b), a large thermal anomaly remains and the impactor's core is buried within the martian mantle (c). Then, this dense metallic material rapidly sinks towards the pre-impact martian core. During the sinking, potential energy is converted as heat in the mantle via viscous dissipation. Two sinking regimes occur (c and e) depending on the distance $h$ between the impactor and the martian core (see section 4). Finally the intruded metallic material merges with the preexisting core, spreads at its top (e) and eventually mixes with it later (f) (which we don't model here). The hatched volumes represent the metallic material from the core and the impactor. 


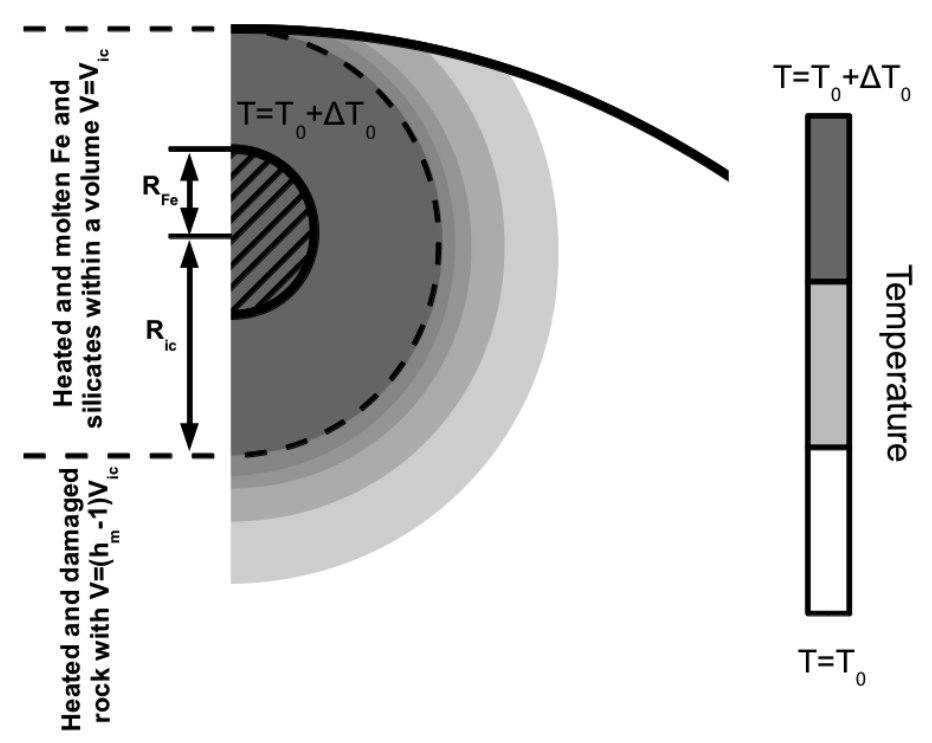

Figure 2: Sketch to show repartitioning of the post impact heating in the martian mantle. The solid black line represents the planetary surface. Melting is restricted to the material limited by the dashed line. The impactor's core is represented hatched lines. 

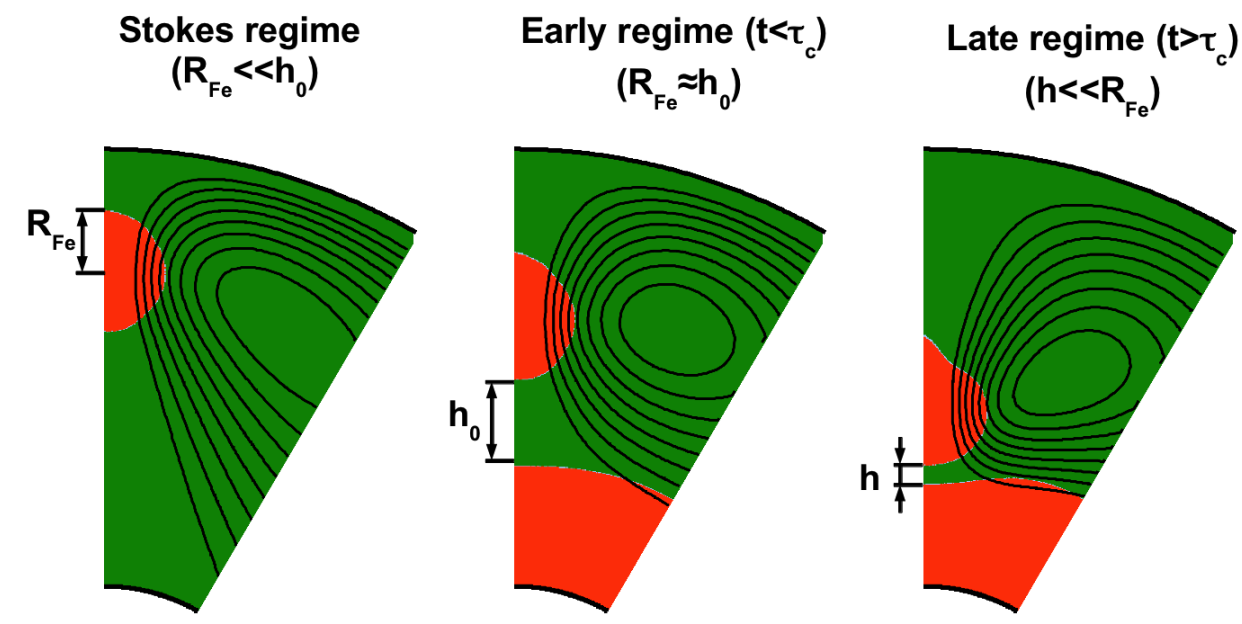

Figure 3: Dynamics of core merging for the three regimes described in section 4.3 and computed for $R_{F e}=300 \mathrm{~km}$ sinking in an isoviscous differentiated Mars size planet (silicate material is represent in green and metallic material is represented in red). Solid black lines illustrate the streamlines resulting from the diapir sinking. In the left figure, where we do not consider the presence of a planetary core, the viscous stress scales with $1 / R_{F e}$. In the middle figure, interaction with the core start to matter and the viscous stress scales with $1 / h_{0}$. In the right figure where $h<R_{F e}$, interactions between the diapir and the merging core increase and the lubrication force scales with $1 / h$. 


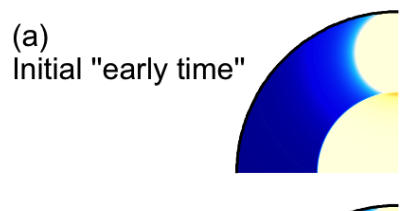

(b)

"Late time"
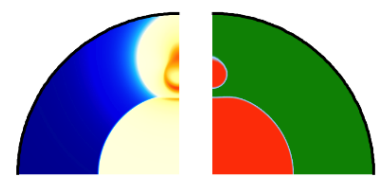

(c)

Merging phase
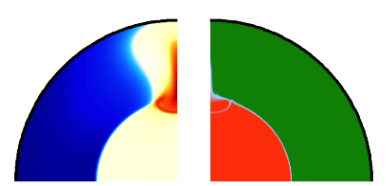

(d)
Spreading phase
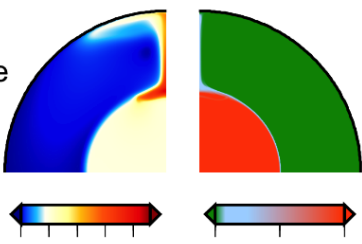

01234

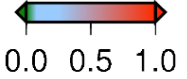

Figure 4: Non-dimensional temperature (left) and composition (right) at times $\mathrm{t}=0$ (a), $\mathrm{t}$ $=0.6 \mathrm{Myr}(\mathrm{b}), \mathrm{t}=6 \mathrm{Myr}(\mathrm{c})$ and $\mathrm{t}=38.8 \mathrm{Myr}(\mathrm{d})$ (computed for a uniform viscosity with $R=3400 \mathrm{~km}, R_{i m p}=600 \mathrm{~km}$ and $300 \times 600$ grid points). As the diapir sinks, its velocity decreases because of the increasing influence of the pre-existing core on the dynamics (see section 4.3 for details). After the merging (c), the hot metallic fraction from the impactor spreads at the top of the martian core $(d)$. 


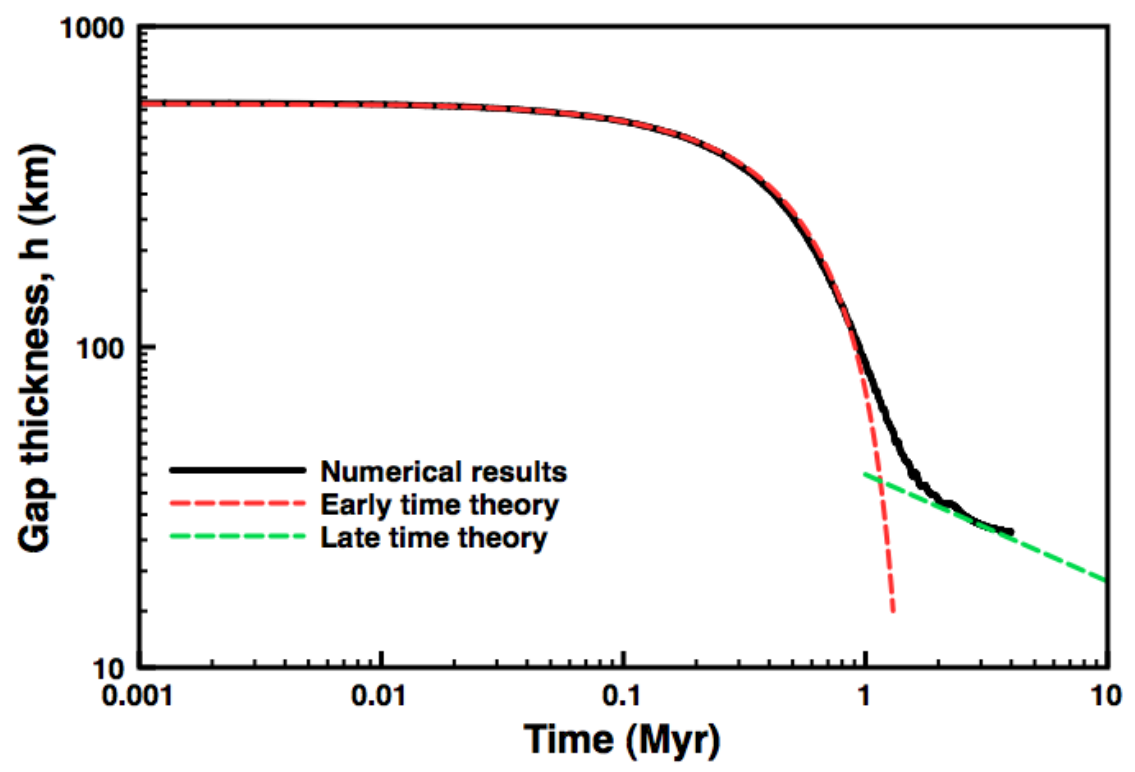

Figure 5: Evolution of the gap thickness between the merging core and the CMB with $R_{i m p}=$ $600 \mathrm{~km}$ (i.e. $R_{\mathrm{Fe}}=300 \mathrm{~km}$ ). Theoretical evolutions for early times from Eq.12 and late times from Eq.14 are shown with red and green dashed lines respectively. From this model, we obtain a coalescence time, $t_{c}=1.55$, Myr and $a_{2} \sim B o=1.3 \times 10^{9}$. 


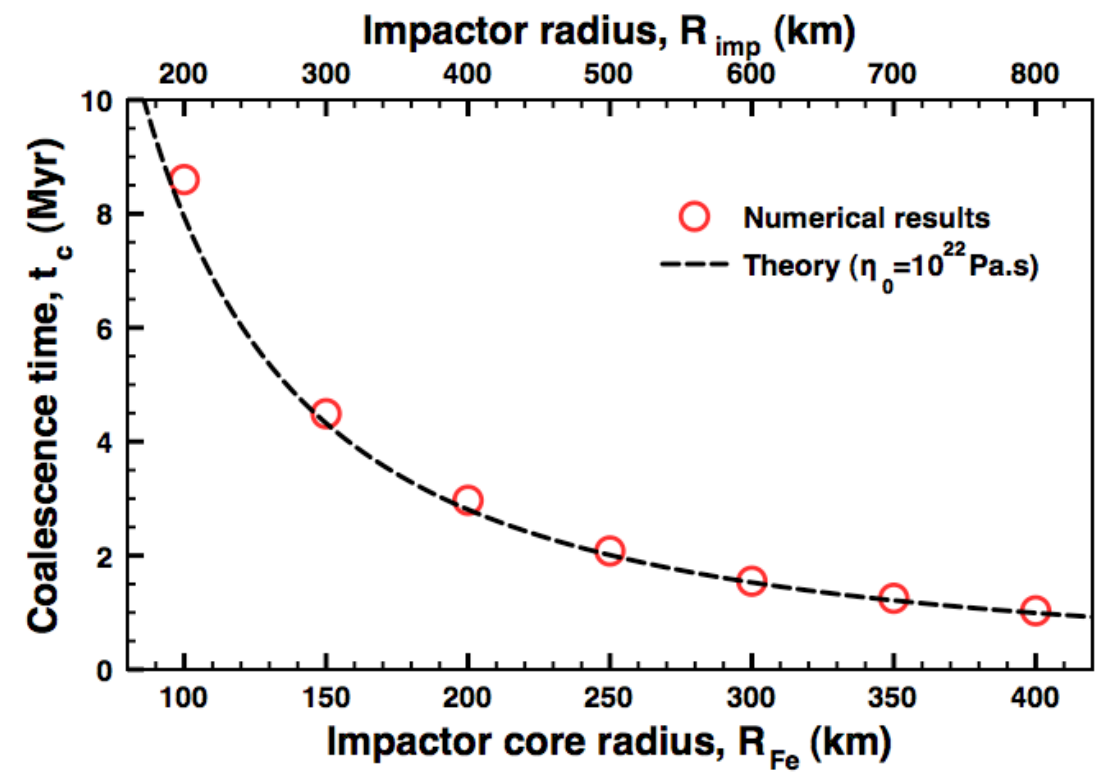

Figure 6: Characteristic coalescence time, $t_{c}$, as a function of the impactor core radius $R_{F e}$. Results from numerical experiments (with uniform viscosity $\eta_{0}=10^{22} \mathrm{~Pa} . \mathrm{s}$ and $h_{0}=580 \mathrm{~km}$ ) are represented with red circles. Theoretical fit from Eq.13 is shown with the black dashed line $\left(a_{1}=1 / 9\right)$ 


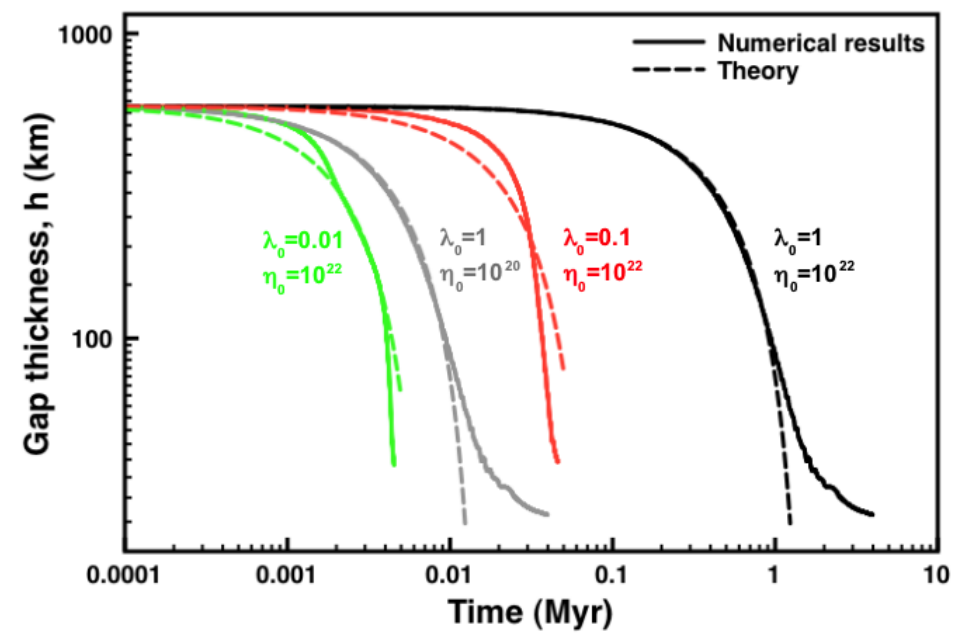

Figure 7: Time evolution of the gap thickness between the sinking core and the pre-existing core for four different rheologies. Solid black line and solid grey line represent results from uniform viscosity models with $\eta_{0}=10^{22}$ and $\eta_{0}=10^{20}$ respectively. Solid red line and solid green line represent results from temperature dependent viscosities with $\lambda=0.1$ and $\lambda=0.01$ respectively (with $\eta=\eta_{0} \lambda^{T}$ and $\eta_{0}=10^{22} \mathrm{~Pa} . \mathrm{s}$ ). Theoretical predictions are shown in the corresponding color with dashed lines for the coalescence theory (Eq.12) or with dotted lines for the Rybczynski-Hadamard theory (Eq.9). 

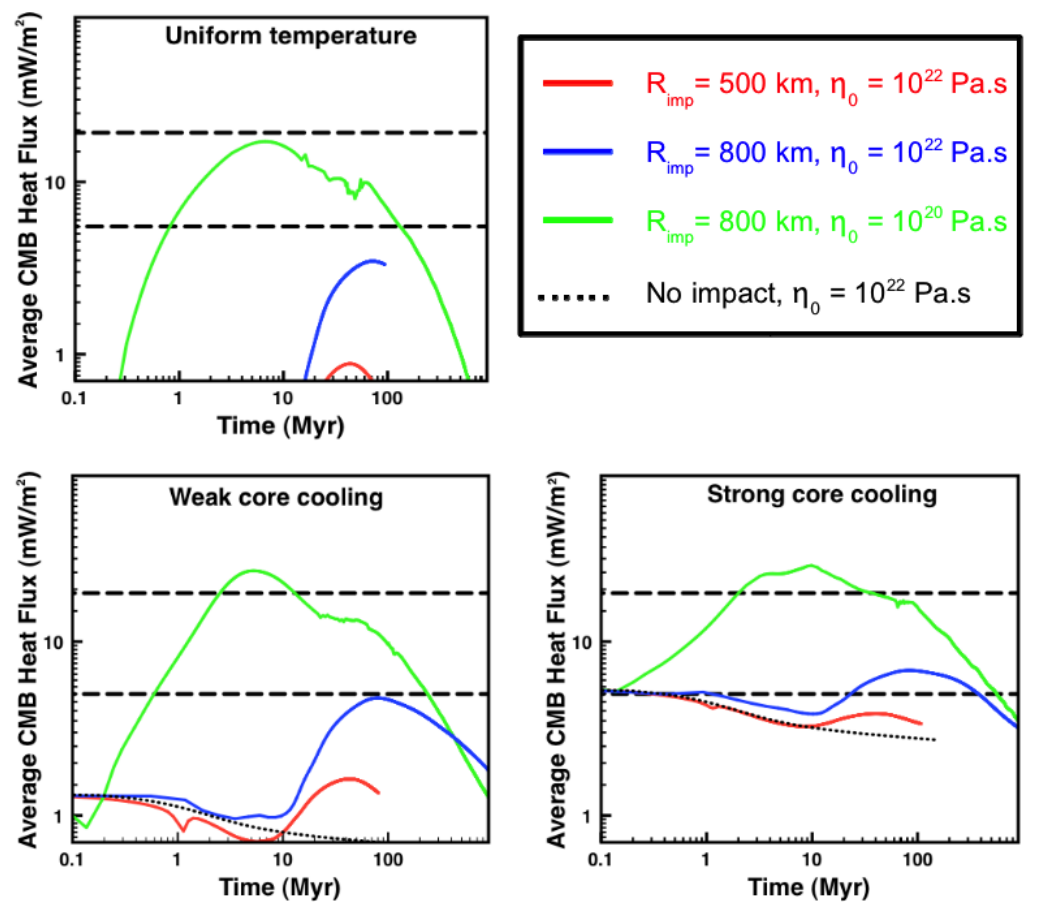

Figure 8: Time evolution of the mean CMB heat flux as a function of the rheology and impactor size. In the three panels different initial thermal states before impact are considered. The two horizontal black dashed lines represent the theoretical dynamo criterion for $q_{C M B}$ $\left(q_{C M B}>q_{A}=5-19 \mathrm{~mW} \cdot \mathrm{m}^{-2}\right)$. In the top-left panel, pre-impact temperature is assumed to be constant $\left(T=T_{0}\right)$. In the bottom panels, the core is initially hotter than the mantle $T=T_{\text {core }}>T_{0}$. In the bottom left panel the CMB thermal boundary layer thickness $\delta=0.4 R_{F e}$ and in the bottom right panel $\delta=0.1 R_{F e}$. Red, blue and green solid lines represent the evolution with $\left(R_{i m p}=500 \mathrm{~km}, \eta_{0}=10^{22}\right.$ Pa.s. $),\left(R_{i m p}=800 \mathrm{~km}, \eta_{0}=10^{22}\right.$ Pa.s. $)$, and $\left(R_{i m p}=800 \mathrm{~km}, \eta_{0}=10^{20}\right.$ Pa.s. $)$ respectively. The black dotted line represent $q_{C M B}$ without any impact. 